\title{
PHASE DIAGRAMS OF ONE-DIMENSIONAL MODELS UNDER STRONG RANDOM EXTERNAL FIELD
}

\author{
AZER KERIMOV \\ Department of Mathematics, Bilkent University, 06533 Bilkent, Ankara, Turkey \\ kerimov@fen.bilkent.edu.tr
}

Received 27 September 2002

\begin{abstract}
We consider one-dimensional models of classical statistical physics and prove that at each fixed value of the temperature for all realizations of additional sufficiently strong random external field the limiting Gibbs state is unique.
\end{abstract}

\section{Introduction}

It is well-known that in one-dimensional models the phenomenon of phase transition essentially depends on the decay rate of the potential: in models with a pair potential $U(|x-y|)$ satisfying the condition

$$
\sum_{y \in \mathbf{Z}^{1}}|x-y| U(|x-y|)<\infty
$$

the phase transition is absent. ${ }^{1-3}$ Other models certainly may exhibit a phase transition; for example, the one-dimensional ferromagnetical Ising model with the Hamiltonian

$$
H(\phi)=-\sum_{x, y \in \mathbf{Z}^{1}}|x-y|^{-1-\alpha} \phi(x) \phi(y)
$$

where spin variables $\phi(x), \phi(y)$ take values 1 and -1 and $0<\alpha<1$ at low temperatures, has at least two limiting Gibbs states corresponding to the constant ground states $\phi=1$ and $\phi=-1 .^{4,5}$

In this paper we investigate one-dimensional models of classical statistical physics without specifying the interaction potential and prove rather a natural result: at any fixed value of the temperature and under sufficiently strong random external field the set of all the limiting Gibbs states has at most one element.

Let us consider a model on $\mathbf{Z}^{1}$ with the formal Hamiltonian

$$
H_{0}(\phi)=\sum_{B \subset \mathbf{Z}^{1}} U(\phi(B))
$$


where the spin variables $\phi(x) \in \Phi, \Phi$ is a finite subset of the real line $\mathbf{R}, \phi(B)$ denotes the restriction of the configuration $\phi$ to the set $B$, on which the potential $U(\phi(B))$ is not necessarily translationally invariant.

On the potential $U(\phi(B))$ we impose a natural condition, necessary for the existence of the thermodynamic limit:

$$
\sum_{B \subset \mathbf{Z}^{1}: x \in B}|U(\phi(B))|<C_{0}
$$

where the constant $C_{0}$ does not depend on $x$ and the configuration $\phi$.

Now we consider random perturbations of the model (1), namely a model with the Hamiltonian

$$
H(\phi)=H_{0}(\phi)+\sum_{x \in \mathbf{Z}}^{1} h_{x} \phi(x)
$$

where $\left\{h_{x}, x \in \mathbf{Z}^{1}\right\}$ is a random external field.

The main result of the present paper is the following: ${ }^{\mathrm{a}}$

Theorem 1. For any model (1) and any fixed value of the inverse temperature $\beta$ there exists a constant $h_{0}$ such that for all realizations of the random external field $\left\{h_{x}, x \in \mathbf{Z}^{1}\right\}$ satisfying $\left|h_{x}\right|>h_{0}, x \in \mathbf{Z}^{1}$ the model (3) has atmost one limiting Gibbs state.

Let $\mathbf{P}^{1}$ and $\mathbf{P}^{2}$ be two extreme limiting Gibbs states corresponding to the boundary conditions $\phi^{1}$ and $\phi^{2}$. It is well known that $\mathbf{P}^{1}$ and $\mathbf{P}^{2}$ are singular or coincide. ${ }^{6,7}$ We prove the uniqueness of the limiting Gibbs states of model (3) by showing that $\mathbf{P}^{1}$ and $\mathbf{P}^{2}$ are not singular.

Let $V_{N}$ be an interval with the center at the origin and with the length of $2 N$. We will denote by $\Phi(N)$ the set of all configurations $\phi\left(V_{N}\right)$. Suppose that the boundary conditions $\phi^{i}, i=1,2$ are fixed.

The concatenation of the configurations $\phi\left(V_{N}\right)$ and $\phi^{i}\left(\mathbf{Z}^{1}-V_{N}\right)$ we denote by $\chi: \chi(x)=\phi(x)$, if $x \in V_{N}$ and $\chi(x)=\phi^{i}(x)$, if $x \in \mathbf{Z}^{1}-V_{N}$. Let us define

$$
H_{N}\left(\phi \mid \phi^{i}\right)=\sum_{B \subset \mathbf{Z}^{\nu}: B \cap V_{N} \neq \emptyset} U(\chi(B)) .
$$

If the expression $\left|H_{N}\left(\phi \mid \phi^{i}\right)\right|$ is bounded uniformly with respect to $N, \phi$ and $\phi^{i}$ then the non-singularity of $\mathbf{P}^{1}$ and $\mathbf{P}^{2}$ directly follows. This simple but rather useful idea was first used in Ref. 5 for the proof of the absence of phase transition in one-dimensional models with long-range interaction. But in our more general case $\left|H_{N}\left(\phi \mid \phi^{i}\right)\right|$ need not to be bounded and we use more sophisticated approach.

Due to Lemma 1 below the configuration with minimal energy at fixed $N$ and boundary conditions $\phi^{i}$ is unique and independent of $\phi^{i}$ if $\left|h_{x}\right|>h_{0}, x \in \mathbf{Z}^{1}$, where $h_{0}$ is sufficiently large:

$$
\min _{\phi \in \Phi(N)} H_{N}\left(\phi \mid \phi^{i}\right)=H_{N}\left(\phi_{N}^{\min , i} \mid \phi^{i}\right) \text { where } \phi_{N}^{\min , i}=\phi_{N}^{\min } .
$$

${ }^{\text {a }}$ Later in Section 2 we give the proof. 
Below, $H_{N}\left(\phi \mid \phi^{i}, \phi_{N}^{\min }\right)$ denotes the relative energy of a configuration $\phi$ (with respect to $\left.\phi_{N}^{\min }\right)$ :

$$
H_{N}\left(\phi \mid \phi^{i}, \phi_{N}^{\min }\right)=H_{N}\left(\phi \mid \phi^{i}\right)-H_{N}\left(\phi_{N}^{\min } \mid \phi^{i}\right) .
$$

Let $\mathbf{P}_{\mathbf{N}}^{\mathbf{i}}$ be Gibbs distributions on $\Phi(N)$ corresponding to the boundary conditions $\phi^{i}, i=1,2$ defined using the relative energies of configurations. Take $M<N$ and let $\mathbf{P}_{\mathbf{N}}^{\mathbf{i}}\left(\phi^{\prime}\left(V_{M}\right)\right.$ be the probability of the event that the restriction of the configuration $\phi\left(V_{N}\right)$ to $V_{M}$ coincides with $\phi^{\prime}\left(V_{M}\right)$.

In order to show that $\mathbf{P}^{1}$ and $\mathbf{P}^{2}$ are not singular, we prove that there exist two positive constants const 1 and const $_{2}$, such that for any $M$ and $\phi^{\prime}\left(V_{M}\right)$ there exists a number $N_{0}(M)$ such that for any $N>N_{0}(M)$, we have

$$
\text { const }_{1}<\mathbf{P}_{\mathbf{N}}^{1}\left(\phi^{\prime}\left(V_{M}\right)\right) / \mathbf{P}_{\mathbf{N}}^{2}\left(\phi^{\prime}\left(V_{M}\right)\right)<\text { const }_{2} .
$$

The first important point is the introduction of the contour model common for boundary conditions $\phi^{i}, i=1,2$ (a contour is a connected subconfiguration not coinciding with the ground state). After that, using a well-known trick ${ }^{8}$ we come to "noninteracting" clusters from interacting contours (a cluster is a collection of contours connected by interaction bonds).

The second important point is combinatorial Lemma $3,{ }^{9}$ which allows us to reduce the dependence of the expression

$$
\mathbf{P}_{\mathbf{N}}^{1}\left(\phi\left(V_{M}\right)\right) / \mathbf{P}_{\mathbf{N}}^{2}\left(\phi\left(V_{M}\right)\right)
$$

on the boundary conditions $\phi^{1}$ and $\phi^{2}$ to the sum of statistical weights of some 2-clusters connecting the cube $V_{M}$ with the boundary (so-called long 2-clusters; since the statistical weight of 2-cluster is not necessarily positive, we estimate the sum of absolute values of statistical weights of long 2-clusters). Finally it turns out that if the additional random field is strong enough the sum of statistical weights of 2-clusters connecting $V_{M}$ with the boundary is negligible and the expression $\mathbf{P}_{\mathbf{N}}^{1}\left(\phi\left(V_{M}\right)\right) / \mathbf{P}_{\mathbf{N}}^{2}\left(\phi\left(V_{M}\right)\right)$ is bounded.

\section{Proof of Theorem 1}

Let $\varphi_{V}^{\min , 1} \in \Phi(V)$ be a configuration with the minimal energy at fixed boundary conditions $\phi^{1}$. The following simple lemma describes the structure of the configuration $\phi_{V}^{\min , 1}$.

Lemma 1. For any model (3) there exists a positive constant $h_{0}$ such that for all realizations of the random field $\left\{h_{x}, x \in \mathbf{Z}^{1}\right\}$ satisfying $\left|h_{x}\right|>h_{0}$ the configuration $\phi_{V}^{\min , 1}$ is unique and independent of the boundary conditions $\left(\phi^{1}\right)$.

Proof. The lemma is a straightforward consequence of the condition (2).

Let $\mathbf{P}^{1}$ and $\mathbf{P}^{2}$ be two extreme limiting Gibbs states corresponding to the boundary conditions $\phi^{1}$ and $\phi^{2}$. 
Theorem 2. At any fixed value of the inverse temperature $\beta$ there exists a constant $h_{0}$ such that for all realizations of the random external field $\left\{h_{x}, x \in \mathbf{Z}^{1}\right\}$ satisfying $\left|h_{x}\right|>h_{0}, x \in \mathbf{Z}^{1}$, the limiting Gibbs measures $\mathbf{P}^{1}$ and $\mathbf{P}^{2}$ are not singular.

Proof. In order to prove this theorem it is enough to show that there exists two positive constants $c_{1}$ and $C_{1}$ such that for any $M$ and $\phi^{\prime}\left(V_{M}\right)$ we have

$$
c_{1} \leq \mathbf{P}^{1}\left(\phi^{\prime}\left(V_{M}\right)\right) / \mathbf{P}^{2}\left(\phi^{\prime}\left(V_{M}\right)\right) \leq C_{1} .
$$

Since the limiting Gibbs states $\mathbf{P}^{1}$ and $\mathbf{P}^{2}$ are the weak limits of the measures $\mathbf{P}_{\mathbf{N}}^{1}$ and $\mathbf{P}_{\mathbf{N}}^{2}$ when $N \rightarrow \infty$, for establishing (4) we need to prove that there exists two positive constants $c_{1}$ and $C_{1}$ such that for any $M$ and $\phi^{\prime}\left(V_{M}\right)$ there exists a number $N_{0}(M)$ such that for any $N>N_{0}(M)$, we have

$$
c_{1}<\mathbf{P}_{\mathbf{N}}^{1}\left(\phi^{\prime}\left(V_{M}\right)\right) / \mathbf{P}_{\mathbf{N}}^{2}\left(\phi^{\prime}\left(V_{M}\right)\right)<C_{1} .
$$

Suppose that the boundary conditions $\phi^{1}$ are fixed. Consider the probability $\mathbf{P}_{\mathbf{N}}^{1}$ of the event that the restriction of the configuration $\phi\left(V_{N}\right)$ to $V_{M}$ coincides with $\phi^{\prime}\left(V_{M}\right)$ :

$$
\begin{aligned}
& \mathbf{P}_{\mathbf{N}}^{1}\left(\phi^{\prime}\left(V_{M}\right)\right) \\
& =\frac{\sum_{\phi\left(V_{N}\right): \phi\left(V_{M}\right)=\phi^{\prime}\left(V_{M}\right)} \exp \left(-\beta H_{N}\left(\phi\left(V_{N}\right) \mid \phi^{1}, \phi_{N}^{\min , 1}\right)\right)}{\sum_{\phi\left(V_{N}\right)} \exp \left(-\beta H_{N}\left(\phi\left(V_{M}\right) \mid \phi^{1}, \phi_{N}^{\min , 1}\right)\right)} \\
& =\frac{\exp \left(-\beta H_{M}^{\mathrm{in}}\left(\phi^{\prime}\left(V_{M}\right)\right)\right) Y\left(\phi^{\prime}\left(V_{M}\right), V_{N}, \phi^{1}\right) \Xi\left(V_{N}-V_{M} \mid \phi^{1}, \phi^{\prime}\left(V_{M}\right), \phi_{N}^{\min , 1}\right)}{\sum_{\phi^{\prime \prime}\left(V_{M}\right)} \exp \left(-\beta H_{M}^{\text {in }}\left(\phi^{\prime \prime}\left(V_{M}\right)\right) Y\left(\phi^{\prime \prime}\left(V_{M}\right), V_{N}, \phi^{1}\right) \Xi\left(V_{N}-V_{M} \mid \phi^{1}, \phi^{\prime \prime}\left(V_{M}\right), \phi_{N}^{\min , 1}\right)\right.} \\
& =\frac{\exp \left(-\beta H_{M}^{\mathrm{in}}\left(\phi^{\prime}\left(V_{M}\right)\right) Y\left(\phi^{\prime}\left(V_{M}\right), V_{N}, \phi^{1}\right) \Xi^{\phi^{1}, \phi^{\prime}}\right.}{\sum_{\phi^{\prime \prime}\left(V_{M}\right)} \exp \left(-\beta H_{M}^{\mathrm{in}}\left(\phi^{\prime \prime}\left(V_{M}\right)\right) Y\left(\phi^{\prime \prime}\left(V_{M}\right), V_{N}, \phi^{1}\right) \Xi^{\phi^{1}, \phi^{\prime \prime}}\right.}
\end{aligned}
$$

where the summation in $\sum_{\phi^{\prime \prime}\left(V_{M}\right)}$ is taken over all possible configurations of $\phi^{\prime \prime}\left(V_{M}\right)$. Hence

$$
H_{M}^{\mathrm{in}}\left(\phi^{\prime}\left(V_{M}\right)\right)=\sum_{B \subset V_{M}} U\left(\phi^{\prime}(B)\right)-U\left(\phi_{N}^{\min , 1}\right)
$$

and

$$
H_{M}^{\mathrm{in}}\left(\phi^{\prime \prime}\left(V_{M}\right)\right)=\sum_{B \subset V_{M}} U\left(\phi^{\prime \prime}(B)\right)-U\left(\phi_{N}^{\min , 1}\right)
$$

are interior relative energies of $\phi^{\prime}\left(V_{M}\right)$ and $\phi^{\prime \prime}\left(V_{M}\right)$. $\Xi^{\phi^{1}, \phi^{\prime}}$ and $\Xi^{\phi^{1}, \phi^{\prime \prime}}$ denote the partition functions corresponding to the boundary conditions $\phi^{1}\left(\mathbf{Z}^{1}-\right.$ $\left.V_{N}\right), \phi^{\prime}\left(V_{M}\right), \phi^{\prime \prime}\left(V_{M}\right)$ :

$$
\begin{aligned}
& \Xi^{\phi^{1}, \phi^{\prime}}=\Xi\left(V_{N}-V_{M} \mid \phi^{1}, \phi^{\prime}\left(V_{M}\right), \phi_{N}^{\min , 1}\right), \\
& \Xi^{\phi^{1}, \phi^{\prime \prime}}=\Xi\left(V_{N}-V_{M} \mid \phi^{1}, \phi^{\prime \prime}\left(V_{M}\right), \phi_{N}^{\min , 1}\right) .
\end{aligned}
$$


The factor $Y\left(\phi\left(V_{M}\right), V_{N}, \phi^{1}\right)$ is defined as

$$
Y\left(\phi\left(V_{M}\right), V_{N}, \phi^{1}\right)=\prod_{\substack{A \subset \mathbf{Z}^{1}: A \cap V_{M} \neq \emptyset ; \\ A \cap \mathbf{Z}^{1}-V_{N} \neq \emptyset ; \\ A \cap V_{N}-V_{M}=\emptyset}} \exp \left(-\beta\left(U(\phi(A))-U\left(\phi_{N}^{\min , 1}(A)\right)\right)\right)
$$

where $\phi$ in Eq. (8) is equal to $\phi^{\prime}$ for $x \in V_{M}$ and is equal to $\phi^{1}$ for $x \in \mathbf{Z}^{1}-V_{N}$.

The expression (8) gives the "direct" interaction of $\phi\left(V_{M}\right)$ with the boundary conditions $\phi^{1}\left(\mathbf{Z}^{1}-V_{N}\right)$. The probability $\mathbf{P}_{\mathbf{V}}^{1}\left(\varphi^{\prime}\left(V_{M}\right)\right)$ is given by Eq. (6). We can express $\mathbf{P}_{\mathbf{V}}^{2}\left(\varphi^{\prime}\left(V_{M}\right)\right)$ in just the same way. (10):

In order to prove the inequality (5) it is enough to establish inequalities (9) and

$$
0.9<Y\left(\phi\left(V_{M}\right), V_{N}, \varphi^{i}\right)<1.1, \quad i=1,2
$$

and

$$
1 / S_{2} \leq\left(\frac{\Xi^{\phi^{1}, \phi^{\prime \prime}}}{\Xi^{\phi^{1}, \phi^{\prime}}}\right) /\left(\frac{\Xi^{\phi^{2}, \phi^{\prime \prime}}}{\Xi^{\phi^{2}, \phi^{\prime}}}\right) \leq 1 / s_{2}
$$

for arbitrary $\varphi^{\prime \prime}\left(V_{M}\right)$, where $S_{2}=(1.1 / 0.9)^{2} S$ and $s_{2}=(0.9 / 1.1)^{2} s_{1}$.

Indeed, if the inequalities (9) and (10) hold, then

$$
1 /\left(1 / s_{1}\right) \leq \mathbf{P}_{\mathbf{V}}^{1}\left(\varphi^{\prime}\left(V_{M}\right)\right) / \mathbf{P}_{\mathbf{V}}^{2}\left(\varphi^{\prime}\left(V_{M}\right)\right) \leq 1 /\left(1 / S_{1}\right)
$$

since the quotient of $\left(\sum_{i=1}^{n} a_{i}\right) /\left(\sum_{i=1}^{n} b_{i}\right)$ lies between $\min \left(a_{i} / b_{i}\right)$ and $\max \left(a_{i} / b_{i}\right)$.

Now we start to prove the inequalities (9) and (10).

The inequality (9) is a direct consequence of the condition that the potential is a decreasing function: For each fixed $M$ there exists $N_{0}$, such that if $N>N_{0}$, then $0.9<Y\left(\phi\left(V_{M}\right), V_{N}, \phi^{i}\right)<1.1$ for $i=1,2$.

So, in order to complete the proof of Theorem 2 we have to establish the following inequality [which is just the transformed inequality (10)]:

$$
\frac{1}{S_{2}} \leq \frac{\Xi^{\phi^{1}, \phi^{\prime \prime}} \Xi^{\phi^{2}, \phi^{\prime}}}{\Xi^{\phi^{2}, \phi^{\prime \prime}} \Xi^{\phi^{1}, \phi^{\prime}}} \leq \frac{1}{s_{2}} .
$$

Now we show that for each fixed interval $V_{M}$, there exists a number $N_{0}(M)$, which depends on $M$ only, such that if $N>N_{0}(M)$

$$
s_{2} \leq \frac{\Xi^{\phi^{1}, \phi^{\prime}} \Xi^{\phi^{2}, \phi^{\prime \prime}}}{\Xi^{\phi^{1}, \phi^{\prime \prime}} \Xi^{\phi^{2}, \phi^{\prime}}} \leq S_{2}
$$

for two positive constants $s_{2}$ and $S_{2}$ independent of $M, \phi^{1}, \phi^{2}, \phi^{\prime}$ and $\phi^{\prime \prime}$.

Let us consider the partition functions

$$
\Xi^{\phi^{1}, \phi^{\prime \prime}}=\Xi\left(V_{N}-V_{M} \mid \phi^{1}, \phi^{\prime \prime}\left(V_{M}\right), \phi_{N}^{\min , 1}\right)
$$


corresponding to the boundary conditions $\phi^{1}\left(\mathbf{Z}^{1}-V_{N}\right), \phi^{\prime \prime}\left(V_{M}\right)$ and

$$
\Xi^{\phi^{2}, \phi^{\prime}}=\Xi\left(V_{N}-V_{M} \mid \phi^{2}, \phi^{\prime}\left(V_{M}\right), \phi_{N}^{\min , 2}\right)
$$

corresponding to the boundary conditions $\phi^{2}\left(\mathbf{Z}^{1}-V_{N}\right), \phi^{\prime}\left(V_{M}\right)$ as in Eq. (7).

Now define a super partition function

$$
\begin{aligned}
& \left(\Xi^{\phi^{1}, \phi^{\prime \prime}} \Xi^{\phi^{2}, \phi^{\prime}}\right) \\
& \quad=\sum \exp \left(-\beta H_{N}\left(\phi^{3}\left(V_{N}\right) \mid \phi^{1}, \phi^{\prime \prime}, \phi_{V}^{\min , 1}\right)\right) \exp \left(-\beta H_{N}\left(\phi^{4}\left(V_{N}\right) \mid \phi^{2}, \phi^{\prime}, \phi_{N}^{\min , 2}\right)\right)
\end{aligned}
$$

where the summation is taken over all pairs of configurations $\phi^{3}\left(V_{N}\right)$ and $\phi^{4}\left(V_{N}\right)$, such that $\phi^{3}\left(V_{M}\right)=\phi^{\prime \prime}\left(V_{M}\right), \phi^{4}\left(V_{M}\right)=\phi^{\prime}\left(V_{M}\right)$.

Consider the partition of $\mathbf{Z}^{1}$ into $V_{x}$ which is an interval with the length of edge 1 and with the center at $x=1 / 2+k$ ( $k$ is an integer). A configuration $\phi^{g r}$ is said to be a ground state of the model (3) if

$$
H\left(\bar{\phi}^{g r}\right)-H\left(\phi^{g r}\right) \geq 0
$$

for all finite perturbations $\phi^{g r}$ (the set $\left\{x: \bar{\phi}^{g r}(x) \neq \phi^{g r}(x)\right\}$ should be finite) of the configuration $\phi^{g r}$. Due to Lemma 1 if $h_{0}$ is sufficiently large and $\left|h_{x}\right|>h_{0}$, the model (3) has a unique ground state $\phi^{g r}$.

Let us consider an arbitrary configuration $\phi$. We say that a cube $V_{x}$ is not regular, if $\phi\left(V_{x}\right) \neq \phi^{g r}\left(V_{x}\right)$. Two non-regular cubes are called connected provided their intersection is not empty. The connected components of non-regular segments defined in such a way are called supports of contours and are denoted by $\operatorname{supp}(K)$. A pair $K=(\operatorname{supp}(K), \phi(\operatorname{supp}(K)))$ is called a contour. Obviously for each contour $K$, there exists a configuration $\psi_{K}$ such that the only contour of the configuration $\psi_{K}$ is $K\left(\psi_{K}\right.$ on $\mathbf{Z}^{1}-\operatorname{supp}(K)$ coincides with $\left.\phi^{g r}\right)$.

Let us define the weight of contour $K$ by the formula:

$$
\gamma(K)=H\left(\psi_{K}\right)-H\left(\phi^{g r}\right) .
$$

The statistical weight of contour $K$ is

$$
w\left(K_{i}\right)=\exp \left(-\beta \gamma\left(K_{i}\right)\right) .
$$

Suppose that the contours of the configuration $\phi\left(V_{N}\right)$ are $K_{1}, \ldots, K_{n}$. The value of the interaction of contours $K_{1}, \ldots, K_{n}$ between themselves and with the boundary conditions $\phi^{1}$ we denote by $G\left(K_{1}, \ldots, K_{n}\right)$. This expression naturally decomposes into the interaction of single contours with the boundary conditions, pairs of contours between themselves and with the boundary conditions, and so on:

$$
G\left(K_{1}, \ldots, K_{n}\right)=\sum_{k=1}^{n} \sum_{K_{i_{1}}, \ldots, K_{i_{k}}} G\left(K_{i_{1}}, \ldots, K_{i_{k}}\right)
$$

(at each fixed $k$ the summation is taken over all possible non-ordered collections $\left.K_{i_{1}}, \ldots, K_{i_{k}}\right)$ and

$$
G\left(K_{i_{1}}, \ldots, K_{i_{k}}\right)=\sum_{B}\left(U(\phi(B))-U\left(\phi^{g r}(B)\right)\right)
$$


where the summation is taken over all $B \subset \mathbf{Z}^{1}$ such that $B \cap \operatorname{supp}\left(K_{i_{j}}\right) \neq \emptyset$ for all $j=1, \ldots, k$. We say that $B$ is an interaction element $\lambda=\lambda\left(i_{1}, \ldots, i_{k}\right)$ corresponding to the the term $G\left(K_{i_{1}}, \ldots, K_{i_{k}}\right)$ if $U(\phi(B))-U\left(\phi^{g r}(B)\right) \neq 0$. The set of all interaction elements $\lambda$ corresponding to the terms $G\left(K_{i_{1}}, \ldots, K_{i_{k}}\right)$ in the double sum (15) will be denoted by $I G$.

The following equation is a straightforward consequence of the formulae (14) and (15):

$$
\exp \left(-\beta H_{N}\left(\phi \mid \phi^{1}, \phi_{N}^{\min , 1}\right)=\prod_{i=1}^{n} w\left(K_{i}\right) \exp \left(-\beta G\left(K_{1}, \ldots, K_{n}\right)\right) .\right.
$$

The interaction between $K_{i_{1}}, \ldots, K_{i_{k}}$ arises due to the fact that the weight of the contour $K_{i_{j}}, j=1, \ldots, k$ was calculated under the assumption that the configuration outside $\operatorname{supp}\left(K_{i_{j}}\right)$ coincides with the ground state. Now we can rewrite (16) as:

$$
\begin{aligned}
\exp \left(-\beta H_{N}\left(\phi \mid \phi^{1}, \phi_{N}^{\min , 1}\right)\right)=\prod_{i=1}^{n} w\left(K_{i}\right) \prod_{\lambda \in I G}(\exp (-\beta G(\lambda))) \\
=\prod_{i=1}^{n} w\left(K_{i}\right) \prod_{\lambda \in I G}(1+\exp (-\beta G(\lambda)-1)) .
\end{aligned}
$$

From Eq. (17) we have

$$
\exp \left(-\beta H\left(\phi \mid \phi^{1}, \phi_{N}^{\min , 1}\right)=\sum_{I^{\prime} \subset I} \prod_{i=1}^{n} w\left(K_{i}\right) \prod_{\lambda \in I^{\prime}} g(G(\lambda))\right.
$$

where the summation is taken over all subsets $I^{\prime}$ (including the empty set) of the set $I$, and $g(G(\lambda))=\exp (-\beta G(\lambda))-1$.

Consider an arbitrary term of the sum (18), which corresponds to the subset $I G^{\prime} \subset I G$. Let the interaction element $\lambda \in I G^{\prime}$. Consider the set $\mathbf{K}$ of all contours such that for each contour $K \subset \mathbf{K}$, the set $\operatorname{supp}(K) \cap \lambda$ is nonempty. We call any two contours from $\mathbf{K}$ neighbors in $I G^{\prime}$ interaction. The set of contours $K^{\prime}$ is called connected in $I G^{\prime}$ interaction if for any two contours $K_{p}$ and $K_{q}$ there exists a collection $\left(K_{1}=K_{p}, K_{2}, \ldots, K_{n}=K_{q}\right)$ such that any two contours $K_{i}$ and $K_{i+1}, i=1, \ldots, n-1$, are neighbors.

The pair $D=\left[\left(K_{i}, i=1, \ldots, s\right) ; I G^{\prime}\right]$, where $I G^{\prime}$ is some set of interaction elements, is called a cluster provided there exists a configuration $\phi$ containing all $K_{i} ; i=1, \ldots, s ; I G^{\prime} \subset I G$; and the set $\left(K_{i}, i=1, \ldots, s\right)$ is connected in $I G^{\prime}$ interaction. The statistical weight of a cluster $D$ is defined by the formula

$$
w(D)=\prod_{i=1}^{s} w\left(K_{i}\right) \prod_{\lambda \in I G^{\prime}} g(G(\lambda)) .
$$

Note that $w(D)$ is not necessarily positive.

Two clusters $D_{1}$ and $D_{2}$ are called compatible provided any two contours $K_{1}$ and $K_{2}$ belonging to $D_{1}$ and $D_{2}$, respectively, are compatible. A set of clusters is 
called compatible provided any two clusters of it are compatible. If $D=\left[\left(K_{i}, i=\right.\right.$ $\left.1, \ldots, s) ; I G^{\prime}\right]$, then we say that $K_{i} \in D ; i=1, \ldots, s$. If $\left[D_{1}, \ldots, D_{m}\right]$ is a compatible set of clusters and $\cup_{i=1}^{m} \operatorname{supp}\left(D_{i}\right) \subset V_{N}$, then there exists a configuration $\phi$ which contains this set of clusters. For each configuration $\phi$ we have

$$
\exp \left(-\beta H_{N}\left(\phi \mid \phi^{1}, \phi_{N}^{\min , 1}\right)\right)=\sum_{I G^{\prime} \subset I G} \prod w\left(D_{i}\right)
$$

where the clusters $D_{i}$ are completely determined by the set $I G^{\prime}$. The partition function is

$$
\Xi\left(\phi^{1}\right)=\sum w\left(D_{1}\right) \cdots w\left(D_{m}\right)
$$

where the summation is taken over all non-ordered compatible collections of clusters.

Thus, we come to suitable noninteracting clusters from awkward interacting contours. $^{8}$

The following generalization of the definition of compatibility allows us to represent $\left(\Xi^{\phi^{1}, \phi^{\prime \prime}} \Xi^{\phi^{2}}, \phi^{\prime}\right)$ as a single partition function.

A set of clusters is called 2-compatible provided any of its two parts coming from two Hamiltonians is compatible. In other words, in 2-compatibility an intersection of supports of two clusters coming from different partition functions is allowed.

If $\left[D_{1}, \ldots, D_{m}\right]$ is a 2-compatible set of clusters and $\cup_{i=1}^{m} \operatorname{supp}\left(D_{i}\right) \subset V_{N}-V_{M}$, then there exist two configurations $\phi^{3}$ and $\phi^{4}$ which contain this set of clusters. For each pair of configurations $\phi^{3}$ and $\phi^{4}$ we have

$$
\exp \left(-\beta H_{N}\left(\phi^{3} \mid \phi^{1}, \phi_{N}^{\min , 1}\right) \exp \left(-\beta H_{N}\left(\phi^{4} \mid \phi^{2}, \phi_{N}^{\min , 2}\right)=\sum_{I G^{\prime} \subset I G, I G^{\prime \prime} \subset I G} \prod w\left(D_{i}\right)\right.\right.
$$

where the clusters $D_{i}$ are completely determined by the sets $I G^{\prime}$ and $I G^{\prime \prime}$. The double partition function is

$$
\Xi^{\phi^{1}, \phi^{\prime \prime}, \phi^{2}, \phi^{\prime}}=\Xi^{\phi^{1}, \phi^{\prime \prime}} \Xi^{\phi^{2}, \phi^{\prime}}=\sum w\left(D_{1}\right) \cdots w\left(D_{m}\right)
$$

where the summation is taken over all non-ordered 2-compatible collections of clusters.

Let $w\left(D_{1}\right) \cdots w\left(D_{m}\right)$ be a term of the double partition function $\Xi^{\phi^{1}, \phi^{\prime \prime}, \phi^{2}, \phi^{\prime}}$. The connected components of the collection $\left[\operatorname{supp}\left(D_{1}\right), \ldots, \operatorname{supp}\left(D_{m}\right)\right]$ are the supports of the superclusters. A supercluster $S D$ is a pair $(\operatorname{supp}(S D), \phi(\operatorname{supp}(S D))$.

A 2-cluster $S D=\left[\left(D_{i}, i=1, \ldots, m\right) ; I G^{\prime}, I G^{\prime \prime}\right]$ is said to be long if the intersection of the set $\left(\cup_{i=1}^{m} \operatorname{supp}\left(D_{i}\right)\right) \cup I G^{\prime} \cup I G^{\prime \prime}$ with both $V_{M}$ and $\mathbf{Z}^{1}-V_{N}$ is non-empty. In other words, a long 2-cluster, by use of its contours and bonds, connects the boundary with the interval $V_{M}$. A set of 2-clusters is called compatible provided the set of all clusters belonging to these 2-clusters are 2-compatible.

Lemma 2. There exists a number $\epsilon(0<\epsilon<1)$ such that for each fixed interval $V_{M}$, there exists a number $N_{0}=N_{0}(M)$, which depends on $M$ only such that if $N>N_{0}$ then 


$$
\begin{aligned}
& (1-\epsilon) \Xi^{\phi^{1}, \phi^{\prime}, \phi^{2}, \phi^{\prime \prime}}<\Xi^{\phi^{1}, \phi^{\prime}, \phi^{2}, \phi^{\prime \prime},(n . l .)} \\
& \quad=\sum w\left(S D_{1}\right) \cdots w\left(S D_{m}\right)<(1+\epsilon) \Xi^{\phi^{1}, \phi^{\prime}, \phi^{2}, \phi^{\prime \prime}}
\end{aligned}
$$

where the summation is taken over all non-long, non-ordered compatible collections of 2-clusters $\left[S D_{1}, \ldots, S D_{m}\right], \cup_{i=1}^{m} \operatorname{supp}\left(S D_{i}\right) \subset V_{N}-V_{M}$ corresponding to the boundary conditions $\left\{\phi^{1}\left(\mathbf{Z}^{1}-V_{N}\right), \phi^{2}\left(\mathbf{Z}^{1}-V_{N}\right) ; \phi^{\prime}\left(V_{M}\right)\right.$ and $\left.\phi^{\prime \prime}\left(V_{M}\right)\right\}$.

In other words, in models with not-long 2-clusters property the statistical weights of long 2-clusters are negligible.

Proof. Let us define a partition function $\Xi^{\phi^{1}, \phi^{\prime}, \phi^{2}, \phi^{\prime \prime},(l .)}$ as $\sum w\left(S D_{1}\right) \cdots w\left(S D_{m}\right)$ where the summation is taken over all terms of $\Xi^{\phi^{1}, \phi^{\prime}, \phi^{2}, \phi^{\prime \prime}}$, which are not included into $\Xi^{\phi^{1}, \phi^{\prime}, \phi^{2}, \phi^{\prime \prime},(n . l .)}$. By dividing of both sides of the equality

$$
\Xi^{\phi^{1}, \phi^{\prime}, \phi^{2}, \phi^{\prime \prime}}=\Xi^{\phi^{1}, \phi^{\prime}, \phi^{2}, \phi^{\prime \prime},(n . l .)}+\Xi^{\phi^{1}, \phi^{\prime}, \phi^{2}, \phi^{\prime \prime},(l)}
$$

by $\Xi^{\phi^{1}, \phi^{\prime}, \phi^{2}, \phi^{\prime \prime}}$, we get

$$
1=\frac{\Xi^{\phi^{1}, \phi^{\prime}, \phi^{2}, \phi^{\prime \prime},(n . l .)}}{\Xi^{\phi^{1}, \phi^{\prime}, \phi^{2}, \phi^{\prime \prime}}}+\frac{\Xi^{\phi^{1}, \phi^{\prime}, \phi^{2}, \phi^{\prime \prime},(l)}}{\Xi^{\phi^{1}, \phi^{\prime}, \phi^{2}, \phi^{\prime \prime}}} .
$$

Consider

$$
\frac{\Xi^{\phi^{1}, \phi^{\prime}, \phi^{2}, \phi^{\prime \prime},(l)}}{\Xi^{\phi^{1}, \phi^{\prime}, \phi^{2}, \phi^{\prime \prime}}}=\frac{\Xi^{\phi^{1}, \phi^{\prime}, \phi^{2}, \phi^{\prime \prime},(l)}}{\Xi^{\phi^{1}, \phi^{\prime}, \phi^{2}, \phi^{\prime \prime},(n . l .)}+\Xi^{\phi^{1}, \phi^{\prime}, \phi^{2}, \phi^{\prime \prime},(l .)}} .
$$

If we replace each term belonging to $\Xi^{\phi^{1}, \phi^{\prime}, \phi^{2}, \phi^{\prime \prime},(l .)}$ by its absolute value, then $\Xi^{\phi^{1}, \phi^{\prime}, \phi^{2}, \phi^{\prime \prime},(l .)}$ turns to $\Xi^{\phi^{1}, \phi^{\prime}, \phi^{2}, \phi^{\prime \prime},(l ., a b s .)}$.

Since the sign of $\Xi^{\phi^{1}, \phi^{\prime}, \phi^{2}, \phi^{\prime \prime},(l .)}$ is not definite, we have (under crucial assumption that $\Xi^{\phi^{1}, \phi^{\prime}, \phi^{2}, \phi^{\prime \prime},(n . l .)}>\Xi^{\phi^{1}, \phi^{\prime}, \phi^{2}, \phi^{\prime \prime},(l ., a b s .)}$, which will follow below from (20)):

$$
\begin{aligned}
& -\frac{\Xi^{\phi^{1}, \phi^{\prime}, \phi^{2}, \phi^{\prime \prime},(l ., a b s .)}}{\left(\Xi^{\phi^{1}, \phi^{\prime}, \phi^{2}, \phi^{\prime \prime},(n . l .)}-\Xi^{\phi^{1}, \phi^{\prime}, \phi^{2}, \phi^{\prime \prime},(l ., a b s .)}\right)} \leq \frac{\Xi^{\phi^{1}, \phi^{\prime}, \phi^{2}, \phi^{\prime \prime},(l .)}}{\left(\Xi^{\phi^{1}, \phi^{\prime}, \phi^{2}, \phi^{\prime \prime},(n . l .)}+\Xi^{\phi^{1}, \phi^{\prime}, \phi^{2}, \phi^{\prime \prime},(l .)}\right)} \\
& \leq \frac{\Xi^{\phi^{1}, \phi^{\prime}, \phi^{2}, \phi^{\prime \prime},(l ., a b s .)}}{\left(\Xi^{\phi^{1}, \phi^{\prime}, \phi^{2}, \phi^{\prime \prime},(n . l .)}+\Xi^{\phi^{1}, \phi^{\prime}, \phi^{2}, \phi^{\prime \prime},(l ., a b s .)}\right)} .
\end{aligned}
$$

It can be easily shown that the inequality (19) follows from the following inequality:

$$
\frac{\Xi^{\phi^{1}, \phi^{\prime}, \phi^{2}, \phi^{\prime \prime},(l ., a b s .)}}{\left(\Xi^{\phi^{1}, \phi^{\prime}, \phi^{2}, \phi^{\prime \prime},(n . l .)}+\Xi^{\phi^{1}, \phi^{\prime}, \phi^{2}, \phi^{\prime \prime},(l ., a b s .)}\right)}<\epsilon / 2 .
$$

The expression $\Xi^{\phi^{1}, \phi^{\prime}, \phi^{2}, \phi^{\prime \prime},(l . a b s)} / \Xi^{\phi^{1}, \phi^{\prime}, \phi^{2}, \phi^{\prime \prime}(a b s)}$ naturally can be interpreted as an "absolute probability" of the event that there is at least one long 2-cluster.

Lemma 3. There exists a number $\epsilon(0<\epsilon<1)$ such that for each fixed interval $V_{M}$, there exists a number $N_{0}=N_{0}(M)$, which depends on $M$ only, such that if $N>N_{0}$ then (20) is valid. 
We say that a 2-cluster $S D$ connects configurations the $\phi^{\prime}\left(V_{M}\right)$ and $\phi^{\prime \prime}\left(V_{M}\right)$ with $\phi^{1}$ and $\phi^{2}$ if the support of $S D$ connects $V_{M}$ with $\mathbf{Z}^{1}-V_{M}$. By definitions, supports of long 2-clusters connect $\phi^{\prime}\left(V_{M}\right)$ and $\phi^{\prime \prime}\left(V_{M}\right)$ with $\phi^{1}$ or $\phi^{2}$. In order to prove lemma, it is sufficient to show that at large values of $h_{0}$ the probability that there is at least one 2-cluster connecting $\phi\left(\mathbf{Z}^{1}-V_{M}\right)$ and $\phi\left(V_{M}\right)$ is less than $\epsilon_{1}$, for some $\epsilon_{1}<1$. By definitions, the support of any 2-cluster is the union (connected by interaction elements) of contours or heap of intersected contours some sitting on others. Below we call these contours and heaps of contours 2-contours and denote them by $S K$.

We prove the stronger result asserting that at large values of $h_{0}$ the absolute probability of the event that there is a 2-contour connected to $V_{M}$ by interaction elements is less than $\epsilon_{2}$ for some $\epsilon_{2}<1$. First of all suppose that the support of 2 -cluster $S D$ consists of a simple contour $K$. Then for each $t>0$ there exist a value of the constant $h_{0}$ from Theorem 1 such that

$$
P^{a b s}(K)<\exp (-\beta t|\operatorname{supp}(K)|) .
$$

This is a straightforward consequence of Peierls argument.

Now suppose that the support of 2-cluster $S D$ consists of only 2-contour $S K$ (without interaction elements) including two contours $K_{1}$ and $K_{2}$. We define $|\operatorname{supp}(S K)|=\left|\operatorname{supp}\left(K_{1}\right) \cup \operatorname{supp}\left(K_{2}\right)\right|$ consistently with above definitions. Similarly, for each $s>0$, there exist a value of the constant $h_{0}$ such that

$$
P^{a b s}(S D)<\exp (-\beta s|\operatorname{supp}(S K)|) .
$$

Now we are going to estimate the absolute probability of the event that there is at least one 2-cluster connecting $\phi(-\infty,-N)$ and $\phi^{\prime}\left(V_{M}\right)$. Suppose that the 2-cluster $S D$ is connected to $\phi\left(V_{M}\right)$. Let $S K$ be the 2-contour closest to $V_{M}$ which belong to $S D$ (if there are two we choose one of them). We say that a 2-contour $K^{\prime}$ is a neighbor of the first order of $S K$ and write $S K \leftrightarrow S K^{\prime}$ if $S K$ and $S K^{\prime}$ are connected by interaction element. A 2 -contour $S K^{\prime \prime}$ is called a neighbor of the $q^{\text {th }}$ order of $S K$ provided

$$
S K \leftrightarrow S K_{1} \leftrightarrow S K_{2} \leftrightarrow \cdots \leftrightarrow S K_{q-1} \leftrightarrow S K^{\prime \prime}
$$

and there is no such diagram with fever arrows.

Lemma 4. Let $S K_{0}$ be a 2-contour of order $k$ and suppose that for all 2-contours of order

$$
k+1 w(S K)<\exp \left(-\frac{1}{2} \beta s|\operatorname{supp}(S K)|\right) .
$$

Then

$$
\sum_{S D: S D=\left(S K_{0}, S K, I G^{\prime}, I G^{\prime \prime}\right)} w(S D)<\exp \left(-\frac{1}{2} \beta s\left|\operatorname{supp}\left(S K_{0}\right)\right|\right) .
$$


Lemma 4 states that if we fix a 2-contour and take the summation over all its neighbors then the constant $s$ in statistical weight of this 2-cluster worsens at most to $s / 2$.

Proof. By definitions

$$
\begin{aligned}
& \sum_{S D: S D=}{ }_{\left.S K_{0}, S K, I G^{\prime}, I G^{\prime \prime}\right)} w(S D) \\
= & \sum_{S D: S D=\left(S K_{0}, S K, I G^{\prime}, I G^{\prime \prime}\right)} w\left(S K_{0}\right) w(S K) \prod_{\lambda \in I G^{\prime}, \lambda \in I G^{\prime \prime}} g(G(\lambda))
\end{aligned}
$$

where $g(G(\lambda))=\exp (-\beta G(\lambda))-1$ by $(18)$.

Now we can estimate the right hand side of last equality

$$
\begin{gathered}
\sum_{S D: S D=\left(S K_{0}, S K, I G^{\prime}, I G^{\prime \prime}\right)} w\left(S K_{0}\right) w(S K) \prod_{\lambda \in I G^{\prime}, \lambda \in I G^{\prime \prime}} g(G(\lambda)) \\
\leq w\left(S K_{0}\right) \prod_{x \in \operatorname{supp}\left(S K_{0}\right)}\left(1+\sum_{\lambda: x \in \lambda}|g(G(\lambda))|(1+Q)\right)^{2}
\end{gathered}
$$

where $Q$ is the sum of statistical weights of all 2-contours passing through fixed point: $Q=\sum_{S K: y \in \operatorname{supp}(S K)} w(S K)$. Explanation of formula $(22)$ : interaction element may intersect (or not) any point $x \in \operatorname{supp}\left(S K_{0}\right)$, we have squared the last factor since in 2-contour there are two supports one sitting one the other.

Now note that due to inequality (21) and the fact that the spin space $\Phi$ is finite at sufficiently large value of $s$ we have $Q<1$. Indeed,

$$
\begin{aligned}
Q & =\sum_{m=1}^{\infty} \sum_{S K: y \in \operatorname{supp}(S K),|\operatorname{supp}(S K)|=m} w(S K) \\
& \leq \sum_{m=1}^{\infty} \exp \left(-\frac{1}{2} \beta s m\right) m|\Phi|^{m} \\
& =\sum_{m=1}^{\infty} m \exp \left(\left(-\frac{1}{2} \beta s+\ln |\Phi|\right) m\right) \\
& \leq \sum_{m=1}^{\infty} \exp \left(-\frac{1}{3} \beta s m\right) \leq 1
\end{aligned}
$$

at sufficiently large values of $\beta$. Thus, for any fixed $\beta$ at sufficiently large values of $s$ the expression $Q<1$.

Let us show that $\sum_{\lambda: x \in \lambda}|g(G(\lambda))|$ is finite. Indeed, since by (2),

$$
\sum_{B \subset \mathbf{Z}^{1}: x \in B}|U(\phi(B))|
$$


converges and is uniformly bounded by $C_{0}$, there are finite number of interaction elements $G=U(\phi(B)$ ) (uniformly with respect to configurations $\phi$ ) for which $\beta|G(\lambda)| \geq 1$. Now note that if $\beta|G(\lambda)|<1$ then

$$
g(G(\lambda))=\exp (-\beta G(\lambda))-1<2 \beta|G(\lambda)|
$$

and

$$
\begin{aligned}
\sum_{\lambda: x \in \lambda}|g(G(\lambda))| & =\sum_{\lambda: x \in \lambda: \beta|G(\lambda)| \geq 1}|g(G(\lambda))|+\sum_{G: x \in \lambda: \beta|G(\lambda)|<1}|g(G(\lambda))| \\
& \leq \text { constant }+2 C_{0}=C_{3} .
\end{aligned}
$$

Finally, by Eqs. (22)-(25), we have

$$
\begin{aligned}
\sum_{S D: S D=\left(S K_{0}, S K, I G^{\prime}, I G^{\prime \prime}\right)} w(S D) & \leq w\left(S K_{0}\right) \prod_{x \in \operatorname{supp}\left(S K_{0}\right)}\left(1+2 C_{3}\right)^{2} \\
& =w\left(S K_{0}\right)\left(1+2 C_{3}\right)^{2\left|\operatorname{supp}\left(S K_{0}\right)\right|} \\
& \leq \exp \left(-\beta s\left|\operatorname{supp}\left(S K_{0}\right)\right|\right)\left(1+2 C_{3}\right)^{2\left|\operatorname{supp}\left(S K_{0}\right)\right|} \\
& <\exp \left(-\frac{1}{2} \beta s\left|\operatorname{supp}\left(S K_{0}\right)\right|\right) .
\end{aligned}
$$

Therefore, the proof of Lemma 4 is completed.

By use of Lemma 4 we can estimate the probability of fixed 2-contour $S K_{0}$. Indeed, we consider a super-cluster consisting of $S K_{0}$ and in the first step we fix all 2-contours of order $q-1$ and take the summation over all 2-contours of order $q$, in the second step we fix all 2-contours of order $q-2$ and take the summation over all 2 -contours of order $q-1$, and so on, we repeat this summation process $q-1$ times and get the estimation:

$$
\sum_{S D: S D=\left(S K_{0} \subset S D\right.} w(S D)<\exp \left(-\frac{1}{2} \beta s\left|\operatorname{supp}\left(S K_{0}\right)\right|\right) .
$$

Let $A$ be the event that there is a 2-contour $S K_{0}$ connected to $\phi\left(V_{M}\right)$ by interaction elements. We complete the proof of Lemma 3 by proving that at large values of $s$ the absolute probability $P(A)$ of the event $A$ is less then $\epsilon_{2}$ for some $\epsilon_{2}<1$. Let $\left|\operatorname{supp}\left(S K_{0}\right)\right|=l$ and the distance between $\operatorname{supp}\left(S K_{0}\right)$ and $\left(V_{M}\right)$ is $k$. Then

$$
P(A) \leq \sum_{l=1}^{\infty} \sum_{k=0}^{\infty} \exp \left(-\frac{1}{2} \beta s l\right) \prod g(G(\lambda))
$$

where the product is taken over all interaction elements between $S K_{0}$ and $\phi^{\prime}\left(V_{M}\right)$. Now

$$
P(A) \leq \sum_{l=1}^{\infty} \exp \left(-\frac{1}{2} \beta s l\right) C_{3}^{l}<\sum_{l=1}^{\infty} \exp \left(-\frac{1}{3} \beta s l\right) \leq \exp \left(-\frac{1}{4} \beta s\right)<\epsilon_{2}<1
$$


if $\beta s$ is sufficiently large $\left(C_{3}\right.$ is a constant defined in $\left.(25)\right)$.

Lemma 3 is proved. As pointed out the inequality (20) implies the inequalities (19), thus the proof of Lemma 2 is also completed.

Partition functions including only non-long super clusters satisfy the following key lemma which has a geometrically-combinatorial explanation.

Lemma 5. ${ }^{9,10}$

$$
\Xi^{\phi^{1}, \phi^{\prime \prime}, \phi^{2}, \phi^{\prime},(n . l .)}=\Xi^{\phi^{1}, \phi^{\prime}, \phi^{2}, \phi^{\prime \prime},(n . l .)} .
$$

Proof. The summations in $\Xi^{\phi^{1}, \phi^{\prime \prime}, \phi^{2}, \phi^{\prime},(n . l .)}=\Xi^{\phi^{1}, \phi^{\prime}, \phi^{2}, \phi^{\prime \prime},(n . l .)}$ are taken over all non-long, non-ordered compatible collections of 2-clusters.

We put a one-to-one correspondence between the terms of these two double partition functions.

Figure 1 shows how this one-to-one correspondence can be carried out. To the term

$$
w\left(D_{1}^{1,,^{\prime \prime}}\right) w\left(D_{2}^{1,{ }^{\prime \prime}}\right) w\left(D_{3}^{1,,^{\prime \prime}}\right) w\left(D_{4}^{1,,^{\prime \prime}}\right) w\left(D_{5}^{2,,^{\prime}}\right) w\left(D_{6}^{2,{ }^{\prime}}\right) w\left(D_{7}^{2,{ }^{\prime}}\right) w\left(D_{8}^{2,{ }^{\prime}}\right)
$$

(the first four factors of this term came from the partition function $\Xi^{\phi^{1}, \phi^{\prime \prime}}$ and the last four factors of this term came from the partition function $\left.\Xi^{\phi^{2}}, \phi^{\prime}\right)$ of the super partition function $\Xi^{\phi^{1}, \phi^{\prime \prime}, \phi^{2}, \phi^{\prime},(n . l .)}$, we correspond the term

$$
w\left(D_{1}^{1,{ }^{\prime}}\right) w\left(D_{6}^{1,{ }^{\prime}}\right) w\left(D_{7}^{1,{ }^{\prime}}\right) w\left(D_{4}^{1, \prime^{\prime}}\right) w\left(D _ { 5 } ^ { 2 , , ^ { \prime \prime } } w ( D _ { 2 } ^ { 2 , { } ^ { \prime \prime } } ) w \left(D_{3}^{2,,^{\prime \prime}} w\left(D_{8}^{2,,^{\prime \prime}}\right)\right.\right.
$$

(the first four factors of this term came from the partition function $\Xi^{\phi^{1}, \phi^{\prime}}$ and the last four factors of this term came from the partition function $\Xi^{\phi^{2}, \phi^{\prime \prime}}$ ) of the super

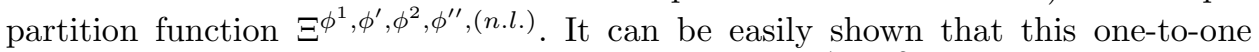
correspondence is well defined: if some term from $\Xi^{\phi^{1}, \phi^{\prime}, \phi^{2}, \phi^{\prime \prime},(n . l .)}$ corresponding to the term from $\Xi^{\phi^{1}, \phi^{\prime \prime}, \phi^{2}, \phi^{\prime},(n . l \text {.) }}$ does not exist (in other words, the corresponding clusters from $\Xi^{\phi^{1}, \phi^{\prime}}$ or $\Xi^{\phi^{2}, \phi^{\prime \prime}}$ are overlapped) then the term from $\Xi^{\phi^{1}, \phi^{\prime \prime}, \phi^{2}, \phi^{\prime},(n . l .)}$ is long super cluster, a contradiction. Therefore, Lemma 5 is proved.

The inequality (12) is a direct consequence of (19) and Lemma 5. The proof of Theorem 2 is completed.

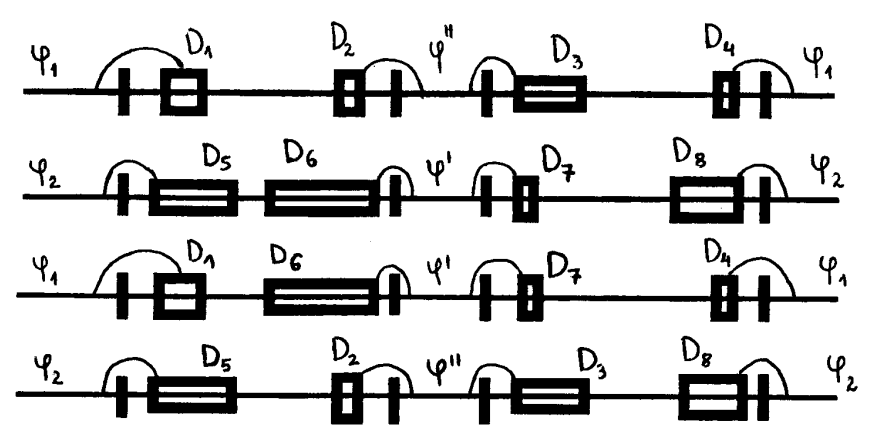

Fig. 1. 
Let $\mathbf{P}^{1}$ and $\mathbf{P}^{2}$ be two extreme limit Gibbs states corresponding to the boundary conditions $\phi^{1}$ and $\phi^{2}{ }^{6,7}$

Theorem 3. $\mathbf{P}^{1}$ and $\mathbf{P}^{2}$ are singular or coincide. ${ }^{6,7}$

Proof of Theorem 1. Due to Theorem 2, $\mathbf{P}^{1}$ and $\mathbf{P}^{2}$ are not singular. Thus, by Theorem $3, \mathbf{P}^{1}$ and $\mathbf{P}^{2}$ coincide. Therefore, Theorem 1 is proved.

\section{Acknowledgments}

The author thanks the referees for their useful suggestions.

\section{References}

1. R. L. Dobrushin, Theor. Prob. Appl. 18, 201 (1968).

2. R. L. Dobrushin, Func. Anal. Appl. 2, 44 (1968).

3. D. Ruelle, Comm. Math. Phys. 9, 267 (1968).

4. F. Dyson, Comm. Math. Phys. 12, 91 (1969).

5. F. Dyson, Comm. Math. Phys. 21, 269 (1971).

6. Ya. G. Sinai, Theory of Phase Transitions: Rigorous Results (Oxford, Pergamon Press, 1982).

7. H-O. Georgii, Gibbs Measures and Phase Transitions (de Gruyter, 1988).

8. J. Bricmont, K. Kuroda and J. L. Lebowitz, Commun. Math. Phys. 101, 501 (1985).

9. A. Kerimov, J. Stat. Phys. 72, 571 (1993).

10. A. Kerimov, J. Phys. A35, 5365 (2002). 